\title{
HEC MONTREAAL
}

Institut

d'économie appliquée

Strategic Exploitation of a Common Resource under Environmental Risk

Eric FESSELMEYER

Marc SANTUGINI

Cahier de recherche $n^{\circ}$ IEA-09-08

Version révisée

February 2012 
Institut d'économie appliquée

HEC Montréal

3000 chemin de la Côte-Sainte-Catherine

Montréal (Québec) H3T 2A7

Canada

http://www.hec.ca/iea

iea.info@hec.ca

Copyright (C) 2009 HEC Montréal.

Tous droits réservés pour tous pays. Toute traduction ou toute reproduction sous quelque forme que ce soit est interdite sans l'autorisation expresse de HEC Montréal.

Les textes publiés dans la série des Cahiers de recherche HEC Montréal n'engagent que la responsabilité de leurs auteurs.

ISSN : 0825-8643 


\title{
Strategic Exploitation of a Common Resource under Environmental Risk
}

\author{
Eric Fesselmeyer* Marc Santugini ${ }^{\dagger}$
}

February 24, 2012

*Department of Economics, National University of Singapore. Email: ecsef@nus.edu.sg

†Institute of Applied Economics and CIRPÉE, HEC Montréal, Canada. Email: marc.santugini@hec.ca. 


\begin{abstract}
We study the effect of environmental risk on the extraction of a common resource. Using a dynamic and non-cooperative game in which an environmental event impacts both the renewability (the future quantity) and the quality of the resource, we show that the anticipation of such an event has an ambiguous effect on present extraction and the tragedy of the commons. On the one hand, a risk of a reduction in the renewability induces the agents to extract less in the present. On the other hand, a risk of a deterioration in the quality of the resource induces the agents to extract more in the present. We then establish a negative relation between conservative behavior and the tragedy of the commons. In particular, when environmental risk induces conservation (when the risk of less renewability is more important than the risk of quality deterioration), there is a larger decrease in present harvesting under social planning than in the non-cooperative game, and the tragedy of the commons is worsened. The reason is that in a non-cooperative game agents do not internalize the risk that too much extraction creates for others, and, thus, decrease their own extraction too little. The social planner does internalize the effect of conservation on all agents, and decreases harvesting more than in the non-cooperative game, which reduces the risk for the whole group of agents. This disparity in conservation leads to a worsening of the tragedy of the commons in addition to overexposure to the risk of less renewability in the non-cooperative game.
\end{abstract}

Keywords: Common resource, Conservation, Dynamic games, Environmental risk, Non-cooperative games, Renewable resource exploitation, Stochastic games, Strategic interactions, Tragedy of the Commons, Uncertainty.

JEL Classifications: C72, C73, D43, D90, L13, O13, Q20, Q54. 


\section{Introduction}

Natural common resources face increasing environmental risks as reported in recent scientific studies. The most common prediction is a widespread reduction in the renewability (i.e., the future quantity) of the stock of natural resources. Declining fish stocks (Backlund et al., 2008); a decrease in global water availability (IPPC, 2007); an overall decline in crop yields for global temperature increases above $3{ }^{\circ} \mathrm{C}$ (IPPC, 2007); and a decrease in growth rates of tropical forests (Hopkin, 2007) are just a few examples. There is also scientific evidence regarding the negative effect of climate changes on the quality of natural resources. The US Department of Agriculture reports that an increase in extreme events brought on by climate changes, such as more frequent flooding, will reduce water quality (Backlund et al., 2008). ${ }^{1}$

Complementary to the scientific research, there exists a large literature in economics that asks how economic behavior is altered in anticipation of events that have detrimental effects on natural resources. ${ }^{2}$ While many papers have found conservative behavior in the context of single-agent dynamic models, less is known about the link between conservative behavior and the tragedy of the commons. Specifically, there remains the question of whether the intensity of conservation differs in the non-cooperative outcome and the social planner's solution and if so, what the effect is on the tragedy of the commons. ${ }^{3}$

It is the purpose of this paper to consider whether the tragedy of the commons is reduced or exacerbated in the presence of environmental risk. To that end, we embed environmental risk in a dynamic and non-cooperative

\footnotetext{
${ }^{1}$ Additionally, if we consider the resource at the aggregate level, then a decrease in the variety of species can be interpreted as a decrease in quality. Such a reduction in variety has the potential to be widespread as $30 \%$ of species are at an increasing risk of extinction (Kerr, 2007).

${ }^{2}$ See among others: Reed (1993), Clarke and Reed (1994), Tsur and Zemel (1995, 1996, 1998), Lafforgue (2005), Alvarez and Koskela (2006), Mitra and Roy (2006), and Polasky et al. (2011).

${ }^{3}$ The issue of whether increased uncertainty leads to more or less conservation has also been studied in the context of technology adoption. For instance, Just et al. (2005) considers the adoption of an existing backstop technology in problems of exhaustible resources when the discovery of superior technologies is anticipated. Delay in adopting the backstop technology would cause the resource to be depleted more rapidly.
} 
extraction game à la Levhari and Mirman (1980) and show that strategic interactions are key in explaining the effect of environmental risk on the tragedy of the commons. We focus on two effects of environmental risk consistent with the scientific evidence exposed above: renewability and quality. We consider a purely exogenous risk, i.e., the exploitation of a lone resource has a relatively small effect on regional or global environmental risk. ${ }^{4}$ This is in contrast to a strand of the literature on (single-agent) optimal control which considers the problem of resource management under uncertainty when the agent can reduce the likelihood of the environmental risk (Clarke and Reed (1994), Tsur and Zemel (1995), Tsur and Zemel (1996), Tsur and Zemel (1998)). Specifically, Tsur and Zemel (1995) study the management of groundwater resources at risk of a permanent and catastrophic event (ceasing exploitation activity). ${ }^{5}$ Optimal exploitation is also studied in the context of managing the level of pollution in the case of environmental events (Clarke and Reed (1994), Tsur and Zemel (1996), Tsur and Zemel (1998)). ${ }^{6}$ In all these cases, conservation can occur to prevent or reduce the likelihood of such events. In contrast, by focusing on a completely exogenous event, the change in behavior is solely due to reducing exposure to risk (instead of altering it). ${ }^{7}$ Hence, we are able to provide results about the pure effect of environmental risk, thereby abstracting from any manipulation by the agents. ${ }^{8}$

\footnotetext{
${ }^{4}$ Exogenous uncertainty has also been studied extensively in models of resource extraction under ownership risk or weak property rights (Long (1975), Bohn and Deacon (2000), Laurent-Lucchetti and Santugini (2011)).

${ }^{5}$ In that vein, see Aflaki (2010) for a recent working paper regarding the effect of uncertainty on the tragedy of the commons in a non-dynamic context. In Aflaki (2010), individuals have an effect on the likelihood of the risk, in the sense that too much exploitation destroys the resource.

${ }^{6}$ Clarke and Reed (1994) consider the case in which the likelihood of the environmental change depends on the level of pollution, while Tsur and Zemel $(1996,1998)$ assume that the occurrence of the event depends on the pollution history.

${ }^{7}$ See also Polasky et al. (2011) for a recent study that combines the case of catastrophic stock collapse with changes in the system dynamics (as in our paper) in the context of a single-agent problem. Conservative behavior prevails whether the risk is exogenous or endogenous.

${ }^{8}$ The effect of exogenous uncertainty (not necessarily related to environmental risk) has been studied in single-agent dynamic problems (Mirman and Spulber (1984), Feliz (1993), Epstein (1996), Mitra and Roy (2006)), as well as dynamic games with strategic behavior (Amir (1996), Laukkanen (2003), Antoniadou et al. (2007), Wang and Ewald (2010)). In these studies, the evolution of the stock depends on random shocks, while, in
} 
We first show that when the environment becomes riskier, harvesting behavior is altered in order to reduce exposure to risk. ${ }^{9}$ On the one hand, if environmental changes lead only to a lower renewability, agents reduce their exposure to this type of risk by harvesting less. The substitution toward future harvesting (and, thus, consumption) is due to precautionary motives since saving more allows one to compensate for a possibly less renewable resource and less future availability of the stock. On the other hand, if environmental changes lead only to lower quality, then agents reduce their exposure to risk by harvesting and consuming more in the present so as to face less risk in terms of future utility flows. When both quality and renewability are at risk of being deteriorated, the direction of the effect depends on the relative strength of the two effects. For instance, if the deterioration in quality is small compared to the negative change in renewability, then agents reduce their exposure to risk by harvesting less. In that case, precautionary motives dominate over concerns for lower future (per-unit) utility flows.

In view of our results, the reason for a change in behavior due to environmental risk is solely motivated by a reduction in the exposure to risk (and not manipulation of likelihood of risk as discussed above). In that sense, our framework is close to Lafforgue (2005), which provides such an analysis in the context of a single-agent optimal control problem of resource extraction when there is amenity value for the exploited stock. The effect of uncertainty is shown to be ambiguous as well, and can lead to conservation. However, the overall effect depends on the size of uncertainty and not the type of uncertainty, as in our paper (i.e., quality vs. renewability uncertainty).

After explaining how different types of risk affect harvesting, we turn to the tragedy of the commons. We show that when environmental risk induces conservation (i.e., when the risk of less renewability is more important than the risk of quality deterioration), the presence of the risk leads to a stronger decrease in present harvesting under social planning than in the non-cooperative game. Hence, the ratio between aggregate harvesting

our approach, the source of uncertainty is the timing of an environmental event that leads to permanent changes in the characteristics of the natural resource.

${ }^{9}$ See Alvarez and Koskela (2006) and Reed (1993) for the issue of risk exposure and risk aversion in the context of forest management. 
in the Cournot-Nash outcome and the socially optimal level of harvesting is increased, which makes the tragedy of the commons worse. Although agents choose to harvest less, they do not internalize the risk that too much extraction creates for others, and, thus, decrease their own extraction too little. The social planner, on the other hand, internalizes this effect and decreases harvesting more. This disparity in conservation leads to a worsening of the tragedy of the commons. We also show that when the risk of quality deterioration is the greatest of both risks. a larger stock means more uncertainty regarding future utility flows. In the non-cooperative game, the agents only care about their own exposure to risk without considering the overall risk in terms of future utility flows, which leads to a weaker increase in harvesting relative to the social planner. As a result, the tragedy of the commons is lessened because the agents fail to reduce exposure to risk in a socially optimal way.

The rest of the paper is organized as follows. In Section 2, we present the model. In Section 3, we characterize the Cournot-Nash equilibrium and the benchmark socially optimal solution. We then study the effect of environmental risk on behavior and the tragedy of the commons in Section 4 . Section 5 concludes and suggests possible extensions.

\section{The Model}

In this section, we embed environmental risk in the Great Fish War dynamic game of Levhari and Mirman (1980). We first recall the benchmark set-up and provide an interpretation of the parameters characterizing the resource. We then introduce environmental risk by rendering these parameters stochastic. 


\subsection{Benchmark Set Up: No Environmental Risk}

Consider the Great Fish War dynamic game in which several agents derive utility from the consumption of a common and renewable resource. ${ }^{10}$ Formally, let $y_{t}$ be the stock of the resource available at the beginning of period $t$. If the resource were to go unexploited in period $t$, the stock would evolve at the beginning of period $t+1$ according to the biological rule

$$
y_{t+1}=y_{t}^{\alpha}
$$

where $\alpha \in(0,1]$.

During period $t$, agent $j$ extracts a quantity $c_{j, t}$ from the stock $y_{t}$, which yields utility $u\left(c_{j, t}, \varphi\right)=\varphi \ln c_{j, t}$ with $\varphi>0 .{ }^{11}$ The present consumption of the resource by the $N$ agents has an effect on the future stock. Using (1), the evolution of the resource follows the rule

$$
y_{t+1}=\left(y_{t}-\sum_{j=1}^{N} c_{j, t}\right)^{\alpha}
$$

where a total of $\sum_{j=1}^{N} c_{j, t}$ is consumed in period $t$ and the remaining $y_{t}-$ $\sum_{j=1}^{N} c_{j, t}$ is left to yield the stock $y_{t+1}$ at the beginning of period $t+1$. In the standard set up, both parameters $\varphi$ and $\alpha$ are known and constant over time.

Before embedding environmental risk in the Great Fish War game, we interpret the parameters $\varphi$ and $\alpha$ in the deterministic case. Consider first parameter $\varphi$. Remark 2.1 states that a higher value of $\varphi$ reflects a higher quality of the resource.

Remark 2.1. For $u\left(c_{j, t}, \varphi\right)=\varphi \ln c_{j, t}$, an increase in $\varphi$ unambiguously increases both the utility and the marginal utility of consumption.

\footnotetext{
${ }^{10}$ Pertinent resources include not only the stock of fish in the ocean, but also the stock of wood in the forest, or the stock of potable water in rivers and lakes. Moreover, the resource may refer to the overall industry, e.g., fish can refer to all species of fish, or it may refer to a specific area of the industry, e.g., tuna fishing.

${ }^{11}$ In Levhari and Mirman (1980), the parameter $\varphi$ is normalized to one since a multiplicative term in the utility function has no effect on behavior in the deterministic case. This parameter does play a role when we introduce environmental risk.
} 
Consider next the interpretation of parameter $\alpha$. We show in Appendix A that, for any given path of strictly positive consumption, an increase in $\alpha$ at time $t$ eventually and permanently reduces the availability of the resource. In other words, there exists $\tau>t$ such that $\frac{\partial y_{k}}{\partial \alpha}<0$ for all $k \geq \tau$. Furthermore, when the stock is less than the carrying capacity, i.e., $y<1$, the decrease in the stock is immediate with $\tau=t+1$. Remark 2.2 states the negative effect of $\alpha$ on availability.

Remark 2.2. Given (2), an increase in a eventually and permanently reduces the future availability of an exploited resource.

\subsection{Environmental Risk}

Having interpreted the relevant parameters of the model, we next introduce environmental risk. To that end, resource characteristics $\varphi$ and $\alpha$ (quality and availability) now depend on the state of the environment $s_{t}$ in the following way. For state of the environment $s_{t}$, the resource available at the beginning of period $t$ is determined by

$$
y_{t}=\left(y_{t-1}-\sum_{j=1}^{N} c_{j, t-1}\right)^{\alpha_{s_{t}}}
$$

and extracting $c_{j, t}$ units during period $t$ yields utility $u\left(c_{j, t}, \varphi_{s_{t}}\right)=\varphi_{s_{t}} \ln c_{j, t}$ to agent $j$.

We adopt a simple process for $s_{t}$. There are two possible states: $s_{t} \in$ $\{1,2\}$. State 1 represents the environment prior to an event that alters the natural resource negatively and permanently. State 2 represents the new environment immediately following this event. The probability of a change in resource characteristics is $\rho \in(0,1]$. In other words, if the state of the environment is $s_{t}=1$ in period $t$, then there is a probability $\rho$ of a permanent change in the subsequent period to state $s_{t+1}=2$. Assumption 2.3 holds for the remainder of the paper.

Assumption 2.3. $\operatorname{Pr}\left[s_{t+1}=2 \mid s_{t}=1\right]=\rho$ and $\operatorname{Pr}\left[s_{t+1}=2 \mid s_{t}=2\right]=1$.

Assumption 2.3 implies that a change is inevitable. ${ }^{12}$ We abstract from

\footnotetext{
${ }^{12}$ The probability of being in state 1 in period $t$ is the probability that the characteristics
} 
asymmetric information and learning by assuming that the probability $\rho$ is known. Here, the parameter $\rho$ represents the common beliefs held by the economy regarding the occurrence of an environmental change. Finally, $\rho$ is exogenous: the extraction activity of the resource has a negligible effect on environmental risk. In other words, the agents exploiting the resource influence neither the likelihood of the environmental event nor the nature of the change. One example for which this is a reasonable framework is the potential shutdown of the thermohaline circulation, which would negatively and permanently impact stocks of fish in the North Atlantic Ocean. Fishing, however, has no known effect on this risk. ${ }^{13}$

We now describe the effect of an environmental change on the resource characteristics.

Assumption 2.4. $\varphi_{1} \geq \varphi_{2}$ and $\alpha_{1} \leq \alpha_{2}$ and at least one inequality holds strictly.

Given the interpretation of the parameters discussed previously and in Appendix A, Assumption 2.4 is consistent with the scientific evidence exposed in the introduction. Indeed, the effect of an environmental event on resource characteristics can be two-fold. First, the environmental change may reduce the quality of the resource: $\varphi_{1}>\varphi_{2}$. Second, a change in the environment may decrease the future availability of the resource: $\alpha_{1}<\alpha_{2}{ }^{14}$ Throughout the rest of the paper we consider environmental events which cause just one of these changes but also the more general case in which both changes are possible.

\section{Non-Cooperation vs. Cooperation}

In this section, we consider behavior under both non-cooperation and cooperation. In the subsequent section, we study the effect of environmental risk

of the resource have not changed in the previous $t$ periods, i.e., $\operatorname{Pr}\left[s_{t}=1\right]=(1-\rho)^{t}$. Hence, $\lim _{t \rightarrow \infty} \operatorname{Pr}\left[s_{t}=2\right]=\lim _{t \rightarrow \infty} 1-(1-\rho)^{t}=1$.

${ }^{13}$ We thank a referee for this example.

${ }^{14}$ Note that in this case it is necessary to add the restriction $\alpha_{1}<1$. 
on the two outcomes as well as on the tragedy of the commons (by comparing the two outcomes).

Since we restrict attention to stationary Markovian strategies, the problem is time-independent and the subscript $t$ is dropped hereafter. A hat sign is used instead to mark time. Specifically, $s$ and $\hat{s}$ represent the state of the environment (and, thus, the characteristics of the resource) today and

tomorrow, respectively. Similarly, $y$ and $\hat{y}=\left(y-\sum_{j=1}^{N} c_{j}\right)^{\hat{\alpha}}$ refer to the resource stock today and tomorrow, respectively.

\subsection{Cournot-Nash Equilibrium}

Under non-cooperation, each agent maximizes the expected sum of discounted utilities over consumption. The agents anticipate the effect of their own present consumption decision as well as the effect of the other agents' consumption decisions on the future stock of the resource. Moreover, each agent anticipates the possibility of a permanent change in resource characteristics. Specifically, in the general case, each agent faces uncertainty about the quality of the resource and the growth of the stock at the beginning of period $t+1$. Therefore, given the stock $y$, the stock dynamics (eq. 3), and the stochastic process defined in Assumption 2.3, the value function of agent $i$ when the environment is in state 1 satisfies

$$
\begin{aligned}
V_{1}^{C N}(y)= & \max _{0 \leq c_{i} \leq y-\sum_{j \neq i} c_{j}} \varphi_{1} \ln c_{i}+(1-\rho) \delta V_{1}^{C N}\left(\left(y-c_{i}-\sum_{j \neq i} c_{j}\right)^{\alpha_{1}}\right) \\
& +\rho \delta V_{2}^{C N}\left(\left(y-c_{i}-\sum_{j \neq i} c_{j}\right)^{\alpha_{2}}\right)
\end{aligned}
$$

where $\delta \in(0,1)$ is the discount factor and $V_{s}^{C N}$ is the value function in a Cournot-Nash $(C N)$ environment when the environment is in state $s$.

In eq. (4), agent $i$ anticipates a possible change in the resource characteristics in the beginning of the subsequent period. With probability $1-\rho$, the characteristics remains the same, i.e., $s=\hat{s}=1$, yielding a stock $\hat{y}=$ $\left(y-\sum_{j=1}^{N} c_{j}\right)^{\alpha_{1}}$ of quality $\varphi_{1}$ in the subsequent period. With probability $\rho$, the characteristics change permanently, yielding a stock $\hat{y}=\left(y-\sum_{j=1}^{N} c_{j}\right)^{\alpha_{2}}$ of quality $\varphi_{2}$. Once the environmental change occurs, there are no more 
anticipated changes. In other words,

$$
V_{2}^{C N}(y)=\max _{0 \leq c_{i} \leq y-\sum_{j \neq i} c_{j}} \varphi_{2} \ln c_{i}+\delta V_{2}^{C N}\left(\left(y-c_{i}-\sum_{j \neq i} c_{j}\right)^{\alpha_{2}}\right)
$$

for any level $y>0$ of the stock. ${ }^{15}$

We characterize the agents' equilibrium behavior prior to the environmental change. Proposition 3.1 contains the symmetric Cournot-Nash solution corresponding to eq. (4), denoted by $g^{C N}(y)$. The proof is relegated to Appendix B. ${ }^{16}$

Proposition 3.1. There exists a unique Cournot-Nash equilibrium to (4). In equilibrium, each agent consumes

$$
g^{C N}(y)=\frac{\varphi_{1} y}{N \varphi_{1}+\frac{(1-\rho) \delta \alpha_{1} \varphi_{1}+\rho \frac{\delta \alpha_{2} \varphi_{2}}{1-\delta \alpha_{2}}}{1-(1-\rho) \delta \alpha_{1}}}
$$

We now perform a comparative analysis of the parameters of the model on optimal behavior. Specifically, we study the effects of $\alpha_{1}, \alpha_{2}, \varphi_{1}$, and $\varphi_{2}$ on $g^{C N}(y)$. A discussion of the effect of $\rho$ on optimal behavior is postponed to Section 4. Consider the first-order condition

$$
\frac{\varphi_{1}}{c}=\delta(1-\rho) \frac{\alpha_{1}}{1-(1-\rho) \alpha_{1} \delta} \frac{\varphi_{1}+\delta \rho \frac{\varphi_{2} \alpha_{2}}{1-\alpha_{2} \delta}}{y-N c}+\delta \rho \frac{\frac{\varphi_{2} \alpha_{2}}{1-\alpha_{2} \delta}}{y-N c}
$$

corresponding to eq. (6). Each agent's optimal consumption equates the marginal utility of consumption with the expected marginal utility of nonharvest, i.e., the marginal utility of consuming one unit less of the resource today. The right-hand side of (7) has two components. The first term represents the marginal utility of non-harvest conditional on the resource characteristics remaining the same for at least one more period and taking account of a possible environmental change later on. The second term represents the

\footnotetext{
${ }^{15}$ If $\rho$ were equal to 0 , then expressions (4) and (5) would be identical.

${ }^{16}$ Formally, the stationary Markovian strategy profile $\left\{g^{C N}(y)\right\}_{j=1}^{N}$ is a symmetric Markov-perfect Nash equilibrium.
} 
marginal utility of non-harvest conditional on an environmental change in the subsequent period leading to an irreversible change in the evolution and quality of the resource. Both terms are discounted by $\delta$ and weighted by the probability of each event.

First, consider the renewability parameters in (7). An increase in either $\alpha_{1}$ and $\alpha_{2}$ decreases the renewability of the resource, which increases the expected marginal utility of non-harvest and causes present consumption to decrease. Second, from (7), an increase in the quality parameter prior to the environmental change, $\varphi_{1}$, has a positive effect on both the marginal utility of consumption and the marginal utility of non-harvest (because the environmental change may not occur in the next period). The effect on the marginal utility of consumption is the strongest of the two, which increases present consumption. Finally, an increase in $\varphi_{2}$ unambiguously increases the expected marginal utility of non-harvest in (7) due to the increase in the quality of future consumption, which reduces present consumption. ${ }^{17}$

\subsection{Social Planner's Problem}

We now turn to the social planner's problem. The objective of the social planner is to maximize the discounted sum of all agents' utilities. Unlike an agent in the Cournot-Nash environment, the social planner internalizes the effect of each agent's consumption on the reduction of the common natural resource. Formally, the value function of the social planner satisfies

$$
\begin{aligned}
V_{1}^{S P}(y)= & \max _{c_{1}, \ldots, c_{N}} \sum_{j=1}^{N} \varphi_{1} \ln c_{j}+(1-\rho) \delta V_{1}^{S P}\left(\left(y-\sum_{j=1}^{N} c_{j}\right)^{\alpha_{1}}\right) \\
& +\rho \delta V_{2}^{S P}\left(\left(y-\sum_{j=1}^{N} c_{j}\right)^{\alpha_{2}}\right),
\end{aligned}
$$

subject to $0 \leq \sum_{j=1}^{N} c_{j} \leq y$. Here, $V_{s}^{S P}$ is the social planner's $(S P)$ value function when the environment is in state $s$. The value function after the

\footnotetext{
${ }^{17}$ Note that the effects for $\varphi_{1}$ and $\varphi_{2}$ pull in opposite directions in equal strength, i.e., $\partial g^{C N}(y) / \partial \varphi_{1}+\partial g^{C N}(y) / \partial \varphi_{2}=0$. In other words, if $\varphi \equiv \varphi_{1}=\varphi_{2}$, then $\partial g^{C N}(y) / \partial \varphi=0$. Hence, the multiplicative taste parameter has an impact on optimal behavior only when it depends on the environment.
} 
environmental change satisfies

$$
V_{2}^{S P}(y)=\max _{c_{1}, \ldots, c_{N}} \sum_{j=1}^{N} \varphi_{2} \ln c_{j}+\delta V_{2}^{S P}\left(\left(y-\sum_{j=1}^{N} c_{j}\right)^{\alpha_{2}}\right)
$$

subject to $0 \leq \sum_{j=1}^{N} c_{j} \leq y$, for any $y>0$.

Proposition 3.2 states the per-agent symmetric social planner's solution corresponding to eq. (8), denoted by $g^{S P}(y)$, The proof is relegated to Appendix B.

Proposition 3.2. There exists a unique solution to (8), in which each agent consumes

$$
g^{S P}(y)=\frac{\varphi_{1} y}{N\left(\varphi_{1}+\frac{(1-\rho) \delta \alpha_{1} \varphi_{1}+\rho \frac{\delta \alpha_{2} \varphi_{2}}{1-\delta \alpha_{2}}}{1-(1-\rho) \delta \alpha_{1}}\right)}
$$

We consider again the effect of $\alpha_{1}, \alpha_{2}, \varphi_{1}$, and $\varphi_{2}$ on the consumption of the social planner, $g^{S P}(y)$, using the first-order condition

$$
\frac{\varphi_{1}}{c}=N \delta(1-\rho) \frac{\alpha_{1}}{1-(1-\rho) \alpha_{1} \delta} \frac{\varphi_{1}+\delta \rho \frac{\varphi_{2} \alpha_{2}}{1-\alpha_{2} \delta}}{y-N c}+N \delta \rho \frac{\frac{\varphi_{2} \alpha_{2}}{1-\alpha_{2} \delta}}{y-N c}
$$

corresponding to eq. (10). Similar to (7), the social planner's optimal choice equates the marginal utility of consumption with the marginal utility of nonharvest, which in this case, internalizes the dynamic externality. In particular, notice that expressions (7) and (11) differ by a multiple of $N$ on the right-hand side. Therefore, the directions of the effects of the renewability and quality parameters on consumption under the social planner are the same as in the Cournot-Nash equilibrium discussed at the end of Section 3.1. Lower renewability, i.e., higher $\alpha_{1}$ or $\alpha_{2}$, decreases present consumption. An increase in initial quality, $\varphi_{1}$, increases present consumption, while an increase in quality after the environmental change, $\varphi_{2}$, decreases present consumption. 


\section{The Effect of Environmental Risk on the Tragedy of the Commons}

In this section, we study the effect of environmental risk on the tragedy of the commons. To that end, using Propositions 3.1 and 3.2, we study the effect

of a riskier environment on $\frac{g^{C N}(y)}{g^{S P}(y)}>1$, the ratio between total harvesting in a non-cooperative game and the socially optimal level of harvesting.

In order to clarify the discussion, we proceed in three steps. We first consider the case in which the environmental change leads only to lower renewability, i.e., $\varphi_{1}=\varphi_{2}$ and $\alpha_{1}<\alpha_{2}$. We then consider the case in which only quality deteriorates, i.e., $\varphi_{1}>\varphi_{2}$ and $\alpha_{1}=\alpha_{2}$. Finally, we allow for both types of changes and provide general conditions for which the tragedy of the commons increases or decreases as the environment becomes riskier. In all cases, when the environment becomes riskier, harvesting behavior is altered to reduce exposure to the risk. The direction of these changes depends on the nature of the environmental change. Additionally, strategic interactions render the changes in behavior weaker (relative to the behavior of the social planner), which explains the changes in the magnitude of the tragedy of the commons.

Proposition 4.1 states that, while a higher likelihood of a less renewable resource entails more conservative behavior, it also exacerbates the tragedy of the commons. The proof is relegated to Appendix B.

Proposition 4.1. Suppose that $\varphi_{1}=\varphi_{2}$ and $\alpha_{1}<\alpha_{2}$. Then,

$$
\begin{aligned}
& \text { 1. } \frac{\partial g^{C N}(y)}{\partial \rho}, \frac{\partial g^{S P}(y)}{\partial \rho}<0 \text {, and } \\
& \text { 2. } \frac{g^{C N}(y)}{g^{S P}(y)} \text { is strictly increasing in } \rho \text {. }
\end{aligned}
$$

The first result of Proposition 4.1 states that, if environmental changes lead only to lower renewability, agents reduce their exposure to this type of risk by harvesting less. The substitution toward future harvesting (and, thus, future consumption) is due to precautionary motives since saving more allows one to compensate for a possibly less renewable resource and less 
future availability of the stock. Note that the risk of having a less renewable resource induces more conservation in both the non-cooperative game and under social planning.

The second result in Proposition 4.1 concerns the tragedy of the commons. It states that the ratio of the Cournot-Nash outcome to the social planner's solution increases as the risk of a change in renewability becomes greater. In other words, when environmental risk induces conservation, the tragedy of the commons increases. The reason is as follows. When environmental changes lead only to a lower renewability, agents reduce their exposure to risk by harvesting less. They do not, however, internalize the risk that their extraction creates for others and, thus, decrease their own extraction too little. The social planner does internalize the effect of conservation on all agents, and decreases harvesting more than in the non-cooperative game, which reduces the risk for the whole group of agents. This disparity in conservation leads to a worsening of the tragedy of the commons in addition to overexposure to the risk of less renewability in the non-cooperative game.

Next, we turn to the case in which the only risk is a possible deterioration in quality. Proposition 4.2 states that, while a higher likelihood of a resource yielding lower quality entails less conservative behavior, it also mitigates the tragedy of the commons. The proof is relegated to Appendix B.

Proposition 4.2. Suppose that $\varphi_{1}>\varphi_{2}$ and $\alpha_{1}=\alpha_{2}$. Then,

$$
\begin{aligned}
& \text { 1. } \frac{\partial g^{C N}(y)}{\partial \rho}, \frac{\partial g^{S P}(y)}{\partial \rho}>0 \text {, and } \\
& \text { 2. } \frac{g^{C N}(y)}{g^{S P}(y)} \text { is strictly decreasing in } \rho \text {. }
\end{aligned}
$$

The first result of Proposition 4.2 states that, if environmental changes lead only to lower quality, then agents reduce their exposure to risk by harvesting more in the present so as to face less risk in terms of future utility flows. Indeed, under a risk of quality deterioration, a larger stock implies more uncertainty regarding future utility flows. In that case, harvesting more implies a reduction in risk exposure. 
The second result in Proposition 4.2 concerns the tragedy of the commons. In the non-cooperative game, when the only risk is quality deterioration, each agent engages in more harvesting. As the agents care only about their own exposure to risk and do not consider the overall risk in terms of future utility flows via the size of the stock, harvesting is increased too little relative to the social planner, who internalizes this externality. As a result, the tragedy of the commons is mitigated. While the socially suboptimal reaction in the non-cooperative game (in terms of the level of risk) turns out to be beneficial from the vantage point of the tragedy of the commons, it is still the case that agents in the non-cooperative game are exposed to a level of risk that is too high compared to the socially optimal level of risk.

In view of Propositions 4.1 and 4.2 , the overall effect of both a deterioration in renewability and the quality of the resource, i.e., $\alpha_{1}<\alpha_{2}$ and $\varphi_{1}>\varphi_{2}$, is ambiguous since the two effects pull in opposite directions. Hence, the direction of the effect depends on the relative strength of the two effects. Proposition 4.3 provides a general condition for which the tragedy of the commons increases or decreases as the environment becomes riskier. The proof is relegated to Appendix B.

Proposition 4.3. Suppose that $\varphi_{1}>\varphi_{2}$ and $\alpha_{1}<\alpha_{2}$. Then,

$$
\frac{\alpha_{2} \varphi_{2}}{1-\delta \alpha_{2}}>\frac{\alpha_{1} \varphi_{1}}{1-\delta \alpha_{1}}
$$

implies that

$$
\begin{aligned}
& \text { 1. } \frac{\partial g^{C N}(y)}{\partial \rho}, \frac{\partial g^{S P}(y)}{\partial \rho}<0 \text {, and } \\
& \text { 2. } \frac{g^{C N}(y)}{g^{S P}(y)} \text { is strictly increasing in } \rho \text {. }
\end{aligned}
$$

We now interpret condition (12) by comparing the marginal rate of nonharvest in the first-order condition of the Cournot-Nash game, (7), with the one in the first-order condition of the social-planner's problem, (11), which we denote $M U N H^{C N}$ and $M U N H^{S P}$. First, we note that when condition (12) 
holds, $M U N H^{C N}$ increases in the probability of an environmental change:

$$
\frac{\partial M U N H^{C N}}{\partial \rho}=\frac{\delta}{y-N c} \frac{1-\delta \alpha_{1}}{\left(1-(1-\rho) \delta \alpha_{1}\right)^{2}}\left(\frac{\varphi_{2} \alpha_{2}}{1-\delta \alpha_{2}}-\frac{\varphi_{1} \alpha_{1}}{1-\delta \alpha_{1}}\right)>0
$$

That is, as $\rho$ increases, the marginal utility of non-harvest increases, causing present consumption to decrease. Second, since $M U N H^{S P}=N \times$ $M U N H^{C N}$, any change in Cournot-Nash behavior is magnified under the social planner. In other words, when consumption decreases in the CournotNash equilibrium, it decreases even more under the social planner leading to a worsening of the tragedy of the commons.

We can now interpret condition (12) by examining how the relative values of $\varphi_{1}$ and $\varphi_{2}$ and of $\alpha_{1}$ and $\alpha_{2}$ affect the marginal utilities of non-harvest and the tragedy of the commons. To that end, we rearrange condition (12) as

$$
\underbrace{\frac{\alpha_{2}}{\frac{\alpha_{1}}{1-\delta \alpha_{2}}}}_{\text {renewability }} \times \underbrace{\frac{\varphi_{2}}{\varphi_{1}}}_{\text {quality }}>1
$$

When the value of $\alpha_{2}$ increases relative to $\alpha_{1}$, the renewability ratio increases, and the positive effect of $\rho$ on $M U N H^{C H}$ increases:

$$
\left.\frac{\partial^{2} M U N H^{C H}}{\partial \rho \partial \alpha_{2}}\right|_{\varphi_{1}=\varphi_{2}}>0
$$

In this case, precautionary savings becomes more important to compensate for a lower future stock of the resource, magnifying the negative consumption effect of $\rho$ (Proposition 4.1). When $\varphi_{2}$ increases relative to $\varphi_{1}$, the quality ratio increases, and the negative effect of $\rho$ on $M U N H^{C H}$ decreases:

$$
\left.\frac{\partial^{2} M U N H^{C H}}{\partial \rho \partial \varphi_{2}}\right|_{\alpha_{1}=\alpha_{2}}>0
$$

Here, the risk of postponing harvesting decreases, dampening the negative consumption effect of an increase in $\rho$ (Proposition 4.2). If condition (14) holds, then the risk of less renewability is more important than the risk of quality deterioration leading to a decrease in consumption in the Cournot- 
Nash equilibrium and under the social planner. Again, the effect is magnified in the social planner's problem so, overall, the tragedy of the commons increases.

\section{Final Remarks}

We study the economic behavior of agents who extract a common resource when the renewability and the quality of the resource are at risk of being altered by environmental change. In particular, we show that when the agents engage in conservative behavior, the tragedy of the commons is exacerbated due to strategic interactions.

In order to obtain existence of a Markov-perfect Nash equilibrium, we have relied on the Levhari-Mirman model of resource extraction with strategic interactions. While this specification may seem too restrictive, ${ }^{18}$ our results on conservation in a non-cooperative game are consistent with the results derived from the different functional forms used in the literature described in the introduction. The only difference is that we show that whether conservation occurs or not depends on the source of risk. However, in this aspect, our explanation is not specific to the log utility, or the Cobb-Douglas production function, but rather due, in general, to the motivation to reduce the exposure to risk. When risk concerns quality, a reduction in risk exposure is possible by harvesting more. When risk concerns quantity, harvesting less in order to have a buffer saving yields less risk. Similarly, the link between conservation and the tragedy of the commons does not depend on the functional form of the utility and the production functions. Rather, it is due to the presence of strategic interaction and its effect on conservation. For instance, when there is conservation, strategic interactions lead to free-riding, i.e., each agent conserves less (relative to the social planner) because they can free-ride on the conservation of the other agents.

Finally, we note that our model abstracts from two important aspects. First, we have focused on an exogenous environmental change. Endogenizing

\footnotetext{
${ }^{18}$ For instance, it is unlikely that the payoff from zero extraction is negative infinity.
} 
the probability of a climate change as in Clarke and Reed (1994), Tsur and Zemel $(1995,1996,1998)$ should a priori strengthen the result. If agents could reduce the likelihood of the environmental risk by decreasing present extraction, this extra conservation motive could lead to more free-riding in the non-cooperative game, and, thus, would further increase the tragedy of the commons. Second, we have assumed that society does not display a protection motive for the resource, i.e., there is no amenity value. ${ }^{19}$ Assuming that the stock enters directly into preferences as in Lafforgue (2005) should also alter the effect of environmental risk on the tragedy of the commons.

\footnotetext{
${ }^{19}$ Amenities values are also called the compensation effect, i.e., the marginal utility of consumption increases as the resource becomes scarcer (Michel and Rotillon, 1995).
} 


\section{A Interpretation of $\alpha$}

In this appendix, we study the effect of an increase in the Cobb-Douglas parameter $\alpha \in(0,1)$ on the stock of the resource. Using (2), the sign of the derivative $\partial y_{k} / \partial \alpha$ indicates whether an increase in $\alpha$ in period 0 yields a higher or lower stock in period $k$ for a given path of consumption. Proposition A.1 states that an increase in $\alpha$ eventually and permanently decreases the stock of the resource for any given consumption path. In other words, there exists $\tau>0$ such that $\frac{\partial y_{k}}{\partial \alpha}<0$ for all $k \geq \tau$. Furthermore, when the stock is less than the carrying capacity, i.e., $y<1$, the decrease in the stock is immediate with $\tau=1$.

Proposition A.1. Given a path of strictly positive consumption $\left\{c_{j, t}\right\}_{j=1, \ldots, N ; t=1,2, \ldots}$ and (2), there exists $\tau>0$, such that, for all $k \geq \tau$,

$$
\frac{\partial y_{k}}{\partial \alpha}<0
$$

Proof. Without loss of generality, we rewrite (2) as

$$
y_{t+1}=\left(1-\omega_{t}\right)^{\alpha} y_{t}^{\alpha}
$$

where $\omega_{t} \in(0,1)$ is a given rate of total extraction at time $t .^{20}$ Using (18), the stock of an exploited resource can be further rewritten as ${ }^{21}$

$$
y_{t}=y_{0}^{\alpha^{t}} \prod_{s=0}^{t-1}\left(1-\omega_{s}\right)^{\alpha^{t-s}} .
$$

\footnotetext{
${ }^{20}$ Since $\sum_{j=1}^{N} c_{j, t} \in\left(0, y_{t}\right)$, it follows that, for all $t$, there exists $\omega_{t} \in(0,1)$ such that $\sum_{j=1}^{N} c_{j, t} \equiv \omega_{t} y_{t}$.
${ }^{21}$ From $(18)$,

$$
\begin{aligned}
y_{1}= & \left(y_{0}\left(1-\omega_{0}\right)\right)^{\alpha}, \\
y_{2}= & \left(y_{1}\left(1-\omega_{1}\right)\right)^{\alpha}=y_{0}^{\alpha^{2}}\left(1-\omega_{0}\right)^{\alpha^{2}}\left(1-\omega_{1}\right)^{\alpha}, \\
& \vdots \\
y_{t}= & y_{0}^{\alpha^{t}} \prod_{s=0}^{t-1}\left(1-\omega_{s}\right)^{\alpha^{t-s} .} .
\end{aligned}
$$


Using (22),

$$
\frac{\partial y_{t}}{\partial \alpha}=y_{t}\left(t \alpha^{t-1} \ln y_{0}+\sum_{s=0}^{t-1}(t-s) \alpha^{t-s-1} \ln \left(1-\omega_{s}\right)\right)
$$

There are two cases to consider. First, suppose that $y_{0} \in(0,1]$. Then, from (23), $\frac{\partial y_{t}}{\partial \alpha}<0$ for all $t>0$. Hence, $\tau=1$. Suppose next that $y_{0}>1$. While the first term in the parentheses of (23) is always positive, $t \alpha^{t-1} \downarrow 0$. The second term in the parentheses is negative and decreasing in $t$. Moreover, $\sum_{s=0}^{t-1}(t-s) \alpha^{t-s-1} \ln \left(1-\omega_{s}\right) \rightarrow \kappa$, where $\kappa<\frac{\ln (1-\underline{\omega})}{(1-\alpha)^{2}}<0, \underline{\omega}=\min _{s=0}^{t-1}\left\{\omega_{s}\right\}$. Hence, there exists $\tau>0$, such that, for $k \geq \tau, \frac{\partial y_{k}}{\partial \alpha}<0$.

\section{B Proofs}

Proof of Proposition 3.1. We first solve for (5). Plugging the conjecture ${ }^{22}$ $V_{2}^{C N}(y)=a_{2}^{C N} \ln y+b_{2}^{C N}$ into (5) yields

$$
V_{2}^{C N}(y)=\max _{0 \leq c_{i} \leq y-\sum_{j \neq i} c_{j}} \varphi_{2} \ln c_{i}+\delta \alpha_{2} a_{2}^{C N} \ln \left(y-c_{i}-\sum_{j \neq i} c_{j}\right)+\delta b_{2}^{C N} .
$$

The first-order condition is

$$
\frac{\varphi_{2}}{c_{i}}=\frac{\delta \alpha_{2} a_{2}^{C N}}{y-c_{i}-\sum_{j \neq i}^{N} c_{j}} .
$$

Evaluating $(25)$ at $c_{i}=c_{j}, \forall j \neq i$ yields

$$
c^{* C N}(y)=\varphi_{2} y /\left(N \varphi_{2}+\delta \alpha_{2} a_{2}^{C N}\right) .
$$

\footnotetext{
${ }^{22}$ The conjecture can be inferred by solving the problem recursively as done in Levhari and Mirman (1980). By solving recursively, one realizes that the value function is always linear in $\ln y$. Moreover, the limit of the solution for the $t$-period game as $t$ goes to infinity is the solution to the infinite-horizon game that we consider.
} 
Plugging (26) into (24) yields

$$
\begin{aligned}
V_{2}^{C N}(y) & =\varphi_{2} \ln c^{* C N}(y)+\delta \alpha_{2} a_{2}^{C N} \ln \left(y-N c^{* C N}(y)\right)+\delta b_{2}^{C N} \\
& =\left(\varphi_{2}+\delta \alpha_{2} a_{2}^{C N}\right) \ln y+\varphi_{2} \ln \omega_{2}^{C N}+\delta \alpha_{2} a_{2}^{C N} \ln \left(1-N \omega_{2}^{C N}\right)+\delta b_{2}^{C N}, \\
& \equiv a_{2}^{C N} \ln y+b_{2}^{C N},
\end{aligned}
$$

where $\omega_{2}^{C N}=\varphi_{2} /\left(N \varphi_{2}+\delta \alpha_{2} a_{2}^{C N}\right)$. Imposing consistency, i.e., $\varphi_{2}+\delta \alpha_{2} a_{2}^{C N}=$ $a_{2}^{C N}$ and $\varphi_{2} \ln \omega_{2}^{C N}+\delta \alpha_{2} a_{2}^{C N} \ln \left(1-N \omega_{2}^{C N}\right)+\delta b_{2}^{C N}=b_{2}^{C N}$, yields

$$
a_{2}^{C N}=\frac{\varphi_{2}}{1-\delta \alpha_{2}}
$$

and

$$
b_{2}^{C N}=\frac{\varphi_{2} \ln \omega_{2}^{C N}+\delta \alpha_{2} a_{2}^{C N} \ln \left(1-N \omega_{2}^{C N}\right)}{1-\delta} .
$$

Since both $a_{2}^{C N}$ and $b_{2}^{C N}$ exist, the conjecture is verified. Since the utility and production functions are concave, the solution is unique.

We now solve for (4). Plugging the conjecture $V_{1}^{C N}(y)=a_{1}^{C N} \ln y+b_{1}^{C N}$ and the solution $V_{2}^{C N}(y)=a_{2}^{C N} \ln y+b_{2}^{C N}$ into (4) yields

$$
\begin{aligned}
V_{1}^{C N}(y)= & \max _{0 \leq c_{i} \leq y-\sum_{j \neq i} c_{j}} \varphi_{1} \ln c_{i}+(1-\rho) \delta \alpha_{1} a_{1}^{C N} \ln \left(y-c_{i}-\sum_{j \neq i} c_{j}\right) \\
& +(1-\rho) \delta b_{1}^{C N}+\rho \delta \alpha_{2} a_{2}^{C N} \ln \left(y-c_{i}-\sum_{j \neq i} c_{j}\right)+\rho \delta b_{2}^{C N} .
\end{aligned}
$$

The first-order condition is

$$
\frac{\varphi_{1}}{c_{i}}=\frac{(1-\rho) \delta \alpha_{1} a_{1}^{C N}+\rho \delta \alpha_{2} a_{2}^{C N}}{y-c_{i}-\sum_{j \neq i}^{N} c_{j}},
$$

where $a_{2}^{C N}$ is defined by (30). Evaluating (33) at $c_{i}=c_{j}, \forall j \neq i$ yields the symmetric Cournot-Nash solution

$$
g^{C N}(y)=\frac{\varphi_{1} y}{N \varphi_{1}+\delta\left((1-\rho) \alpha_{1} a_{1}^{C N}+\rho \alpha_{2} a_{2}^{C N}\right)},
$$


where $a_{1}^{C N}$ remains to be solved. Plugging (34) into (32) yields

$$
\begin{aligned}
V_{1}^{C N}(y) & =\left(\varphi_{1}+(1-\rho) \delta \alpha_{1} a_{1}^{C N}+\rho \delta \alpha_{2} a_{2}^{C N}\right) \ln y+\kappa^{C N} \\
& \equiv a_{1}^{C N} \ln y+b_{1}^{C N}
\end{aligned}
$$

where

$\kappa^{C N}=\varphi_{1} \ln \omega_{1}^{C N}+\delta\left((1-\rho) \alpha_{1} a_{1}^{C N}+\rho \alpha_{2} a_{2}^{C N}\right) \ln \left(1-N \omega_{1}^{C N}\right)+(1-\rho) \delta b_{1}^{C N}+\rho \delta b_{2}^{C N}$

and

$$
\omega_{1}^{C N}=\frac{\varphi_{1}}{N \varphi_{1}+\delta\left((1-\rho) \alpha_{1} a_{1}^{C N}+\rho \alpha_{2} a_{2}^{C N}\right)} .
$$

Imposing consistency, i.e., $\varphi_{1}+(1-\rho) \delta \alpha_{1} a_{1}^{C N}+\rho \delta \alpha_{2} a_{2}^{C N}=a_{1}^{C N}$ and $\kappa^{C N}=$ $b_{2}^{C N}$, yields

$$
a_{1}^{C N}=\frac{\varphi_{1}+\rho \delta \alpha_{2} a_{2}^{C N}}{1-(1-\rho) \delta \alpha_{1}}
$$

and

$$
b_{1}^{C N}=\frac{\varphi_{1} \ln \omega_{1}^{C N}+\delta\left((1-\rho) \alpha_{1} a_{1}^{C N}+\rho \alpha_{2} a_{2}^{C N}\right) \ln \left(1-N \omega_{1}^{C N}\right)+\rho \delta b_{2}^{C N}}{1-(1-\rho) \delta},
$$

which verifies the conjecture. Plugging (30) and (39) into (34) yields (6). Since the utility and production functions are concave, the solution is unique.

Proof of Proposition 3.2. This proof follows the same steps as the proof of Proposition 3.1. We provide all steps for the sake of clarity. Plugging the conjecture $V_{2}^{S P}(y)=a_{2}^{S P} \ln y+b_{2}^{S P}$ into (9) yields

$$
V_{2}^{S P}(y)=\max _{c_{1}, \ldots, c_{N}} \sum_{j=1}^{N} \varphi_{2} \ln c_{j}+\delta \alpha_{2} a_{2}^{S P} \ln \left(y-\sum_{j=1}^{N} c_{j}\right)+\delta b_{2}^{S P} .
$$

The first-order conditions are

$$
\frac{\varphi_{2}}{c_{i}}=\frac{\delta \alpha_{2} a_{2}^{S P}}{y-c_{i}-\sum_{j \neq i}^{N} c_{j}}, \quad i=1, \ldots, N
$$


Solving (42) yields the symmetric solution

$$
c^{* S P}(y)=\varphi_{2} y /\left(N \varphi_{2}+\delta \alpha_{2} a_{2}^{S P}\right) .
$$

Plugging (43) into (41) yields

$$
\begin{aligned}
V_{2}^{S P}(y) & =N \varphi_{2} \ln c^{* S P}(y)+\delta \alpha_{2} a_{2}^{S P} \ln \left(y-N c^{* S P}(y)\right)+\delta b_{2}^{S P} \\
& =\left(N \varphi_{2}+\delta \alpha_{2} a_{2}^{S P}\right) \ln y+N \varphi_{2} \ln \omega_{2}^{S P}+\delta \alpha_{2} a_{2}^{S P} \ln \left(1-N \omega_{2}^{S P}\right)+\delta b_{2}^{S P} \\
& \equiv a_{2}^{S P} \ln y+b_{2}^{S P}
\end{aligned}
$$

where $\omega_{2}^{S P}=\varphi_{2} /\left(N \varphi_{2}+\delta \alpha_{2} a_{2}^{S P}\right)$. Imposing consistency, i.e., $N \varphi_{2}+\delta \alpha_{2} a_{2}^{S P}=$ $a_{2}^{S P}$ and $N \varphi_{2} \ln \omega_{2}^{S P}+\delta \alpha_{2} a_{2}^{S P} \ln \left(1-N \omega_{2}^{S P}\right)+\delta b_{2}^{S P}=b_{2}^{S P}$, yields

$$
a_{2}^{S P}=\frac{N \varphi_{2}}{1-\delta \alpha_{2}}
$$

and

$$
b_{2}^{S P}=\frac{N \varphi_{2} \ln \omega_{2}^{S P}+\delta \alpha_{2} a_{2}^{S P} \ln \left(1-N \omega_{2}^{S P}\right)}{1-\delta} .
$$

Since both $a_{2}^{S P}$ and $b_{2}^{S P}$ exist, the conjecture is verified. Since the utility and production functions are concave, the solution is unique.

We now solve for (8). Plugging the conjecture $V_{1}^{S P}(y)=a_{1}^{S P} \ln y+b_{1}^{S P}$ and the solution $V_{2}^{S P}(y)=a_{2}^{S P} \ln y+b_{2}^{S P}$ into (8) yields

$$
\begin{aligned}
V_{1}^{S P}(y)= & \max _{c_{1}, \ldots, c_{N}} \sum_{j=1}^{N} \varphi_{1} \ln c_{j}+(1-\rho) \delta \alpha_{1} a_{1}^{S P} \ln \left(y-\sum_{j=1}^{N} c_{j}\right) \\
& +(1-\rho) \delta b_{1}^{S P}+\rho \delta \alpha_{2} a_{2}^{S P} \ln \left(y-\sum_{j=1}^{N} c_{j}\right)+\rho \delta b_{2}^{S P} .
\end{aligned}
$$

The first-order conditions are

$$
\frac{\varphi_{1}}{c_{i}}=\frac{(1-\rho) \delta \alpha_{1} a_{1}^{S P}+\rho \delta \alpha_{2} a_{2}^{S P}}{y-c_{i}-\sum_{j \neq i}^{N} c_{j}}, \quad i=1, \ldots, N .
$$


where $a_{2}^{S P}$ is defined by (47). Solving (50) yields the symmetric solution

$$
g^{S P}(y)=\frac{\varphi_{1} y}{N \varphi_{1}+\delta\left((1-\rho) \alpha_{1} a_{1}^{S P}+\rho \alpha_{2} a_{2}^{S P}\right)}
$$

where $a_{1}^{S P}$ remains to be solved. Plugging (51) into (49) yields

$$
\begin{aligned}
V_{1}^{S P}(y) & =\left(N \varphi_{1}+(1-\rho) \delta \alpha_{1} a_{1}^{S P}+\rho \delta \alpha_{2} a_{2}^{S P}\right) \ln y+\kappa^{S P} \\
& \equiv a_{1}^{S P} \ln y+b_{1}^{S P}
\end{aligned}
$$

where

$\kappa^{S P}=N \varphi_{1} \ln \omega_{1}^{S P}+\delta\left((1-\rho) \alpha_{1} a_{1}^{S P}+\rho \alpha_{2} a_{2}^{S P}\right) \ln \left(1-N \omega_{1}^{S P}\right)+(1-\rho) \delta b_{1}^{S P}+\rho \delta b_{2}^{S P}$

and

$$
\omega_{1}^{S P}=\frac{\varphi_{1}}{N \varphi_{1}+\delta\left((1-\rho) \alpha_{1} a_{1}^{S P}+\rho \alpha_{2} a_{2}^{S P}\right)} .
$$

Imposing consistency, i.e., $\varphi_{1}+(1-\rho) \delta \alpha_{1} a_{1}^{S P}+\rho \delta \alpha_{2} a_{2}^{S P}=a_{1}^{S P}$ and $\kappa^{S P}=b_{2}^{S P}$, yields

$$
a_{1}^{S P}=\frac{N \varphi_{1}+\rho \delta \alpha_{2} a_{2}^{S P}}{1-(1-\rho) \delta \alpha_{1}}
$$

and

$$
b_{1}^{S P}=\frac{N \varphi_{1} \ln \omega_{1}^{S P}+\delta\left((1-\rho) \alpha_{1} a_{1}^{S P}+\rho \alpha_{2} a_{2}^{S P}\right) \ln \left(1-N \omega_{1}^{S P}\right)+\rho \delta b_{2}^{S P}}{1-(1-\rho) \delta},
$$

which verifies the conjecture. Plugging (47) and (56) into (51) yields (10). Since the utility and production functions are concave, the solution is unique. 
Proof of Propositions 4.1, 4.2, 4.3. Using (6) and (10),

$$
\begin{aligned}
\frac{\partial g^{C N}(y)}{\partial \rho} & =\frac{\varphi_{1} y \Gamma}{\left(N \varphi_{1}+\frac{(1-\rho) \delta \alpha_{1} \varphi_{1}+\rho \frac{\delta \alpha_{2} \varphi_{2}}{1-\delta \alpha_{2}}}{1-(1-\rho) \delta \alpha_{1}}\right)^{2}}, \\
\frac{\partial g^{S P}(y)}{\partial \rho} & =\frac{\varphi_{1} y \Gamma}{\left(\varphi_{1}+\frac{(1-\rho) \delta \alpha_{1} \varphi_{1}+\rho \frac{\delta \alpha_{2} \varphi_{2}}{1-\delta \alpha_{2}}}{1-(1-\rho) \delta \alpha_{1}}\right)^{2}},
\end{aligned}
$$

where

$$
\Gamma=\frac{\delta\left(1-\delta \alpha_{1}\right)\left(\frac{\alpha_{1} \varphi_{1}}{1-\delta \alpha_{1}}-\frac{\alpha_{2} \varphi_{2}}{1-\delta \alpha_{2}}\right)}{\left(1-(1-\rho) \delta \alpha_{1}\right)^{2}}
$$

Furthermore, from (6) and (10),

$$
\frac{g^{C N}(y)}{g^{S P}(y)}=\frac{N \varphi_{1}+N \rho \frac{\delta \alpha_{2} \varphi_{2}}{1-\delta \alpha_{2}}}{N \varphi_{1}-(N-1)(1-\rho) \delta \alpha_{1} \varphi_{1}+\rho \frac{\delta \alpha_{2} \varphi_{2}}{1-\delta \alpha_{2}}}>1
$$

and

$$
\frac{\partial\left(g^{C N}(y) / g^{S P}(y)\right)}{\partial \rho}=\frac{(N-1)\left(1-\delta \alpha_{1}\right) N \delta \varphi_{1}\left(\frac{\alpha_{2} \varphi_{2}}{1-\delta \alpha_{2}}-\frac{\alpha_{1} \varphi_{1}}{1-\delta \alpha_{1}}\right)}{\left(N \varphi_{1}-(N-1)(1-\rho) \delta \alpha_{1} \varphi_{1}+\rho \frac{\delta \alpha_{2} \varphi_{2}}{1-\delta \alpha_{2}}\right)^{2}} .
$$

Hence, from (58), (59), and (62), $\frac{\partial g^{C N}(y)}{\partial \rho}<0, \frac{\partial g^{S P}(y)}{\partial \rho}<0$, and $\frac{g^{C N}(y)}{g^{S P}(y)}$ is strictly increasing in $\rho$ if and only if

$$
\frac{\alpha_{2} \varphi_{2}}{1-\delta \alpha_{2}}>\frac{\alpha_{1} \varphi_{1}}{1-\delta \alpha_{1}}
$$

as stated in Proposition 4.3. Propositions 4.1 and 4.2 are special cases of 
Proposition 4.3. 


\section{References}

S. Aflaki. The Effect of Environmental Uncertainty on the Tragedy of the Commons. INSEAD Working Paper, 2010.

L.H.R. Alvarez and E. Koskela. Does Risk Aversion Accelerate Optimal Forest Rotation Under Uncertainty? J. of Forest Econ., 12(3):171-184, 2006.

R. Amir. Continuous Stochastic Games of Capital Accumulation with Convex Transitions. Games Econ. Behav., 15(2):111-131, 1996.

E. Antoniadou, C. Koulovatianos, and L.J. Mirman. Strategic Exploitation of a Common Property Resource under Uncertainty. Vienna Economic Papers 0703, University of Vienna, Department of Economics, 2007.

P. Backlund, A. Janetos, D.S. Schimel, J. Hatfield, M.G. Ryan, S.R. Archer, and D. Lettenmaier. The Effects of Climate Change on Agriculture, Land Resources, Water Resources, and Biodiversity in the United States. A Report by the U.S. Climate Change Science Program and the Subcommittee on Global Change Research, Washington, D.C., USA, 2008.

H. Bohn and R. T. Deacon. Ownership Risk, Investment, and the Use of Natural Resources. Amer. Econ. Rev., 90(3):526-549, 2000.

H.R. Clarke and W.J. Reed. Consumption/Pollution Tradeoffs in an Environment vulnerable to Pollution-Related catastrophic Collapse. J. Econ. Dynam. Control, 18(5):991-1010, 1994.

G.S. Epstein. The Extraction of Natural Resources from Two Sites under Uncertainty. Econ. Letters, 51(3):309-313, 1996.

R.A. Feliz. The Optimal Extraction Rate of a Natural Resource under Uncertainty. Econ. Letters, 43(2):231-234, 1993.

M. Hopkin. Rising Temperatures Will Stunt Rainforest Growth. Nature, August 2007. 
IPPC. Climate Change 200\%: Synthesis Report. Contribution of Working Groups I, II and III to the Fourth Assessment. (ed. by the Core Writing Team, R.K. Pachauri and A. Reisinger), Intergovernmental Panel on Climate Change, Geneva, Switzerland, 2007.

R.E. Just, S. Netanyahu, and L.J. Olson. Depletion of Natural Resources, Technological Uncertainty, and the Adoption of Technological Subsitutes. Resource Energy Econ., 27(2):91-108, 2005.

R.A. Kerr. Global Warming Is Changing the World. Science, 316(5822): 188-190, 13 April 2007.

G. Lafforgue. Uncertainty and Amenity Values in Renewable Resource Economics. Environ. Resource Econ., 31(3):369-383, 2005.

M. Laukkanen. Cooperative and Non-Cooperative Harvesting in a Stochastic Sequential Fishery. J. Environ. Econ. Manage., 45(2):454-473, 2003.

J. Laurent-Lucchetti and M. Santugini. Ownership Risk and The Use of Common-Pool Natural Resources. Cahiers de recherche 10-03, HEC Montréal, Institut d'économie appliquée, 2011.

D. Levhari and L.J. Mirman. The Great Fish War: An Example Using a Dynamic Cournot-Nash Solution. Bell J. Econ., 11(1):322-334, 1980.

N. V. Long. Resource Extraction under the Uncertainty about Possible Nationalization. J. Econ. Theory, 10(1):42-53, 1975.

P. Michel and G. Rotillon. Disutility of Pollution and Endogenous Growth. Environ. Resource Econ., 6(3):270-300, 1995.

L.J. Mirman and D.F. Spulber. Uncertainty and Markets for Renewable Resources. J. Econ. Dynam. Control, 8(3):239-264, 1984.

T. Mitra and S. Roy. Optimal Exploitation of Renewable Resources under Uncertainty and the Extinction of Species. Econ. Theory, 28(1):1-23, 2006. 
S. Polasky, A. de Zeeuw, and Wagener F. Optimal Management with Potential Regime Shifts. J. Environ. Econ. Manage., 62(2):229-240, 2011.

W.J. Reed. The Decision to Conserve or Harvest Old-growth Forest. Ecolog. Econ., 8(1):45-69, 1993.

Y. Tsur and A. Zemel. Uncertainty and Irreversibility in Groundwater Resource Management. J. Environ. Econ. Manage., 29(2):149-161, 1995.

Y. Tsur and A. Zemel. Accounting for Global Warming Risks: Resource Management Under Event Uncertainty. J. Econ. Dynam. Control, 20(67):1289-1305, 1996.

Y. Tsur and A. Zemel. Pollution Control in an Uncertain Environment. $J$. Econ. Dynam. Control, 22(6):967-975, 1998.

W.K. Wang and C.O. Ewald. A Stochastic Differential Fishery Game for a Two Species Fish Population with Ecological Interaction. J. Econ. Dynam. Control, 34(5):844-857, 2010. 